\title{
$\mathrm{Pd}$ 催化木质素醚类二聚体分子内氢转移断裂 $\mathrm{C}$ - $\mathrm{O}$ 键研究
}

\author{
严龙 ${ }^{a} \quad$ 庞欢 $^{a}$ 黄耀兵 ${ }^{b}$ 傅尧*,a \\ ( ${ }^{a}$ 安徽省生物质洁净能源重点实验室 化学与材料科学学院 中国科学技术大学 合肥 230026) \\ ( ${ }^{b}$ 南京林业大学 化学工程学院 南京 210037)
}

\begin{abstract}
摘要 结合氢转移方法, 研究了木质素模型物 2-(2'-甲氧基苯氧基)-1-苯乙醇(1a)分子在无外加氢源的条件下利用金属 钯催化剂催化发生 $\mathrm{C}-\mathrm{O}$ 键断裂反应. 合成并表征了一系列 Pd 负载型催化剂, 通过优化发现反应体系在环己烷溶剂和 弱碱添加剂 $\mathrm{Na}_{2} \mathrm{HPO}_{4}$ 条件下显示出较好的催化效率. 结合反应特点将催化剂进行改进, 使用 $\mathrm{MgO}$ 作为载体的催化剂 $\mathrm{Pd} / \mathrm{MgO}$ 高效完成了木质素模型物的分子自供氢降解. 反应过程可能分为两步进行：首先，模型物在钯表面先进行脱氢 过程，含羟基的木质素模型物二聚体 1a 脱去氢后生成酮式中间体 2-(2'-甲氧基苯氧基)-1-苯乙酮(1b), 被脱去的氢原子 吸附于钯表面. 随后, 脱氢中间体 $\mathbf{1 b}$ 在 $\mathrm{Pd}$ 催化下与其表面吸附的 $\mathrm{H}$ 作用, 发生催化 $\mathrm{C}-\mathrm{O}$ 键断键过程.
\end{abstract}

关键词 木质素; 解聚; Pd 催化反应; $\mathrm{C}-\mathrm{O}$ 键切断; 氢转移

\section{Supported Pd Catalysts for the C-O Cleavage of the Lignin Derived Model Dimers through Intramolecular Hydrogenolysis Reaction}

\author{
Yan, Long ${ }^{a} \quad{\text { Pang, } \text { Huan }^{a} \quad \text { Huang, Yaobing }}^{b} \quad \mathrm{Fu}, \mathrm{Yao}^{*, a}$ \\ ( ${ }^{a}$ Anhui Province Key Laboratory of Biomass Clean Energy, School of Chemistry and Materials Science, \\ University of Science and Technology of China, Hefei 230026, Anhui, China) \\ ( ${ }^{b}$ College of Chemical Engineering, Nanjing Forestry University, Nanjing 210037, China)
}

\begin{abstract}
The selective depolymerization of the $\mathrm{C}-\mathrm{O}$ ether bonds in lignin has attracted considerable attentions which would facilitate the valorization of lignin. The model compounds with $\beta-O-4$ type ether bonds were the most frequently studied molecules. In this study, we used 2-(2-methoxyphenyl)oxy-1-phenethanol (1a) as model compound for the $\mathrm{C}$ - $\mathrm{O}$ cleavage through intramolecular hydrogenolysis reaction. The hydroxymethyl group acted as the hydrogen source by dehydrogenating over Pd catalysts. Then, the cleavage of $\mathrm{C}-\mathrm{O}$ bond takes place on the supported Pd catalyst with Pd-H active species. All the catalysts were prepared and characterized by XRD, TEM and BET. Among different metal catalysts, Pd catalysts gave best results toward the $\mathrm{C}-\mathrm{O}$ bond cleavage reaction. After that, the influences of solvent were studied and the best solvent was cyclohexane. Different additives such as acids and bases were also tested in the model reaction. The strong base (i.e. $\mathrm{KOH}$ ) showed negative effect on the product yield, but a weak base (i.e. $\mathrm{Na}_{2} \mathrm{HPO}_{4}$ ) gave a promotion effect on the reaction. The result indicated that the modification of the Pd catalysts' supports would be a good option for the $\mathrm{C}-\mathrm{O}$ bond cleavage reaction. Thus, different Pd catalysts with different supporting materials were prepared and tested, and found that $\mathrm{MgO}$, a weak basic support, gave promoted performance on this reaction. Finally, to figure out the reaction pathway, the reaction mixtures with different reaction time were analyzed by GC-MS which showed that the ketone was the main intermediate of the reaction. The result indicated that the main route for the intramolecular hydrogenolysis reaction was (1) the dehydrogenation of $-\mathrm{CCHOH}$ to ketone to form $\mathrm{Pd}-\mathrm{H}$ species and then (2) the $\mathrm{C}-\mathrm{O}$ bond was cleavage by the $\mathrm{Pd}-\mathrm{H}$ species to form the depolymerization products.

Keywords lignin; depolymerization; $\mathrm{Pd}$-catalyzed reaction; $\mathrm{C}-\mathrm{O}$ bond cleavage; catalytic transfer hydrogenation
\end{abstract}

\section{1 引言}

由于煤炭、石油等不可再生资源的快速大量消耗, 随之产生能源危机促使人类对新型可再生能源替代传 统化石资源的需求愈发强烈 ${ }^{[1]}$. 生物质资源作为自然界
储量最大的可再生自然资源越来越受到人们的重视 ${ }^{[2]}$. 而木质纤维素作为生物质资源的重要组成部分, 主要包 含了纤维素、半纤维素和木质素三大部分 ${ }^{[1]}$. 纤维素和 半纤维素主要是六碳糖和五碳糖通过糖苷键链接, 通过 酸水解方式实现糖苷键的解聚，从而获得小分子糖类化

* E-mail: fuyao@ustc.edu.cn

Received May 22, 2014; published August 27, 2014.

Project supported by the National Basic Research Program of China (Nos. 2012CB215306, 2013CB228103), the National Natural Science Foundation of China (No. 21325208, 21361140372, 21172209), the Fundamental Research Funds for the Central Universities (No. WK2060190025), the Specialized Research Fund for the Doctoral Program of Higher Education (No. 20123402130008), Chinese Academy of Sciences (No. KJCX2-EW-J02) and Fok Ying Tung Education Foundation.

项目受 973 计划(Nos. 2012CB215306, 2013CB228103)、国家自然科学基金(Nos. 21325208, 21361140372, 21172209)、教育部中央高校基本科研业务费 专项(No. WK2060190025)、高等学校博士点基金(No. 20123402130008)、中国科学院基金(No. KJCX2-EW-J02)和霍英东教育基金资助. 
合物. 这方面的研究已被广泛报道并取得了许多具有较 高应用价值的成果 ${ }^{[3]}$. 而木质素由于其三维结构复杂、 性质稳定而难于被直接解聚, 因此其发展和利用一直受 到限制.

近些年, 对木质素解聚的研究受到了越来越多的关 注 ${ }^{[4]}$. 在 2D-NMR 现代检测方法帮助下, 证实木质素结 构中的 $\mathrm{C}-\mathrm{O}$ 键键型主要为 4-O-5 型、 $\alpha-\mathrm{O}-4$ 型、 $\beta-\mathrm{O}-4$ 型等 ${ }^{[5 \sim 7]}$, 分别占据了全部醚键的 5\% 10\%、15\% 30\% 和 30\% 50\%. 通过理论计算方法和前人实验得出这三

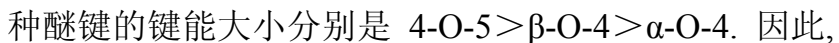
针对这几种不同的醚键类型, 发展高效的催化体系选择 性地解聚这三类醚键对于木质素解聚研究有着重要的意 义.

对于这三类醚键的解聚, Hartwig 等 ${ }^{[8]}$ 报道使用均相 和非均相 Ni 催化剂实现了这些二聚体高选择性地解聚, 获得了相应的酚类和芳环化合物. Lercher 等 ${ }^{[9]}$ 报道使用 负载型的 $\mathrm{Ni}$ 催化剂, 反应对于 $\beta-\mathrm{O}-4$ 和 $\alpha-\mathrm{O}-4$ 两种类型 醚键选择性较高, 而对于 4-O-5 类型的醚键选择性相对 较差, 有过度还原产物生成. 另外, 寇元、徐杰等的研究 组也进行了相关的工作报道 ${ }^{[10 ~ 15]}$. 近来, 新型催化材料 及催化体系也被不断应用于木质素 $\mathrm{C}-\mathrm{O}$ 键切断的工作 并取得一定的成果 ${ }^{[16 ~ 20]}$.

由于 $\beta-O-4$ 型醚键在木质素结构中占有多数 ${ }^{[6]}$, 因 此对该类型的模型物解聚研究受到格外关注 ${ }^{[21,11]}$. Ellman 等 ${ }^{[22]}$ 使用 $\mathrm{RuH}_{2} \mathrm{CO}\left(\mathrm{PPh}_{3}\right)_{2}$ 均相催化剂体系完成了 在无外加氢源条件下的 $\beta-O-4$ 型木质素模型物 2-苯氧 基-1-苯乙醇的 $\mathrm{C}-\mathrm{O}$ 键切断降解(如图 1 所示). 该项工 作为木质素的解聚提供了一条新的思路. Wang 等 ${ }^{[17]}$ 的 研究组也建立了以均相铁为催化中心的催化体系, 在温 和条件下高效完成了上述木质素二聚体模型物的降解 工作. 但是由于均相催化体系自身存在着诸如催化剂难 于回收和反应条件复杂等问题, 可能会制约该催化体系 的放大和实际工业化大规模应用. 因此, 发展绿色高效 的非均相催化体系对于木质素低聚物的解聚有着重要 的意义. 本文主要研究了非均相 $\mathrm{Pd}$ 负载型催化剂在无 外加氢源条件下, 利用 $\beta-O-4$ 型木质素模型物分子自身 羟基作为反应氢源, 实现了其醚键的选择性氢解断裂, 获得了高产率的解聚产物. 同时, 通过对催化剂的表征 和反应条件的优化, 掌握了此类醚类二聚体解聚的反应 规律和反应影响因素, 从而进一步提升了对木质素模型 物分子内供氢反应的认识.

\section{2 结果与讨论}

\section{1 催化剂表征}

我们制备了不同载体负载的 $\mathrm{Pd}$ 催化剂 $\mathrm{Pd} / \mathrm{AC}$ (活性 炭), $\mathrm{Pd} / \mathrm{SiO}_{2}$ 和 $\mathrm{Pd} / \mathrm{MgO}$, 并将这些催化剂和商业化的 $\mathrm{Pd} / \mathrm{C}$ 催化剂一起进行表征对比. TEM 表征结果显示出 四种催化剂上负载的 $\mathrm{Pd}$ 粒子大小及分散性存在区别,
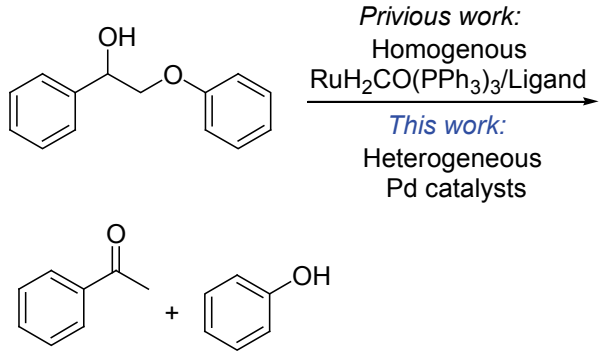

图 1 本文主要研究工作

Figure 1 The main reaction in this research

$\mathrm{Pd} / \mathrm{C}$ 中 $\mathrm{Pd}$ 粒径大约为 $5 \sim 15 \mathrm{~nm} . \mathrm{Pd} / \mathrm{MgO}$ 中 $\mathrm{Pd}$ 粒子粒 径约为 $20 \sim 30 \mathrm{~nm}$, 略大于 $\mathrm{Pd} / \mathrm{C}$ 中 $\mathrm{Pd}$ 的粒径. $\mathrm{Pd} / \mathrm{SiO}_{2}$ 中 $\mathrm{Pd}$ 粒子粒径约为 $30 \sim 40 \mathrm{~nm}$, 而 $\mathrm{Pd} / \mathrm{AC}$ 中 $\mathrm{Pd}$ 粒子的 粒径较大, 达到了约 $40 \mathrm{~nm}$ (如图 2 中所示). 相同条件下 制备的催化剂显示出负载金属颗粒差异可能是由于载 体性质差异导致的. 催化剂中 Pd 粒径大小的区别还在 XRD 表征上得到体现，根据谢乐公式 ${ }^{[23]}$ 可知，衍射峰 强弱与晶粒大小呈正相关. 在 XRD 表征图 3 中显示出 四催化剂中均有 $\mathrm{Pd}$ 的特征衍射峰, 其中 $\mathrm{Pd} / \mathrm{AC} 、 \mathrm{Pd} / \mathrm{SiO}_{2}$ 具有较强的 $\mathrm{Pd}$ 晶粒衍射峰, 而 $\mathrm{Pd} / \mathrm{MgO}$ 相对较弱, $\mathrm{Pd} / \mathrm{C}$ 中的 $\mathrm{Pd}$ 晶体衍射峰相对已经极弱, 这与 TEM 表征一致. 在比表面分析表征中, $\mathrm{Pd} / \mathrm{C}$ 的比表面值为 $871.78 \mathrm{~m}^{2} / \mathrm{g}$, 孔容为 $0.61 \mathrm{~cm}^{3} / \mathrm{g}$, 两参数均低于 $\mathrm{Pd} / \mathrm{AC}$ 的结果 $\left(S_{\mathrm{BET}}=\right.$ $1079.02 \mathrm{~m}^{2} / \mathrm{g}, V_{\mathrm{P}}=1.2 \mathrm{~cm}^{3} / \mathrm{g}$ )(见表 1). $\mathrm{Pd} / \mathrm{SiO}_{2}$ 比表面相 对 $\mathrm{Pd} / \mathrm{C}$ 要低, 只有 $291.0 \mathrm{~m}^{2} / \mathrm{g}$, 但仍远高于 $\mathrm{Pd} / \mathrm{MgO}$ 的 比表面值, $\mathrm{Pd} / \mathrm{MgO}$ 的孔容 $V_{\mathrm{P}}=0.05 \mathrm{~cm}^{3} / \mathrm{g}$, 同样也是四 个对比催化剂中最低的.
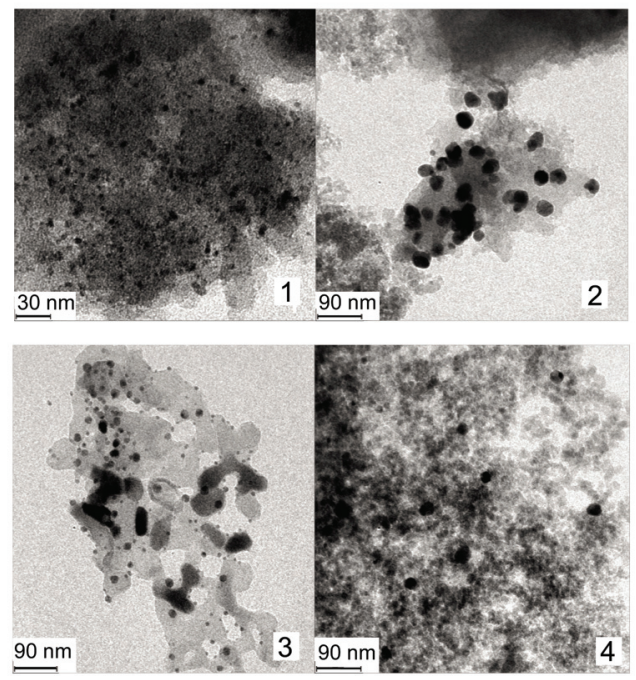

图 2 催化剂 TEM 表征图

Figure 2 TEM images of catalysts

1. $\mathrm{Pd} / \mathrm{C} ; 2$. $\mathrm{Pd} / \mathrm{AC} ; 3$. $\mathrm{Pd} / \mathrm{MgO} ; 4$. $\mathrm{Pd} / \mathrm{SiO}_{2}$

\section{2 反应可行性研究}

我们首先选取了 2-(2'-甲氧基苯氧基)-1-苯乙醇(1a) 作为木质素 $\beta-O-4$ 键型二聚体模型物, 初步选用几种在 


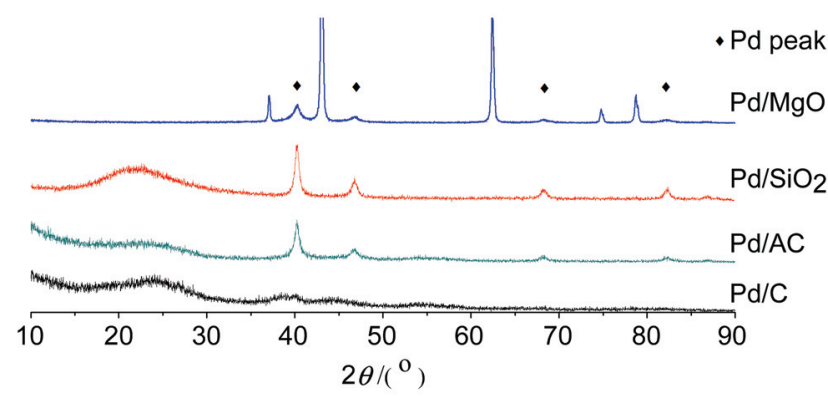

图 3 对比催化剂的 XRD 表征

Figure 3 XRD characteristics of compared catalysts

表 1 催化剂比表面测试结果

Table 1 BET analysis of different catalysts

\begin{tabular}{ccccc}
\hline Entry & Catalyst & $S_{\mathrm{BET}} /\left(\mathrm{m}^{2} \bullet \mathrm{g}^{-1}\right)$ & $D_{\mathrm{p}} / \AA$ & $V_{\mathrm{p}} /\left(\mathrm{cm}^{3} \cdot \mathrm{g}^{-1}\right)$ \\
\hline 1 & $\mathrm{Pd} / \mathrm{C}$ & 871.78 & 28.12 & 0.61 \\
2 & $\mathrm{Pd} / \mathrm{AC}$ & 1079.02 & 44.63 & 1.20 \\
3 & $\mathrm{Pd} / \mathrm{MgO}$ & 17.79 & 110.00 & 0.05 \\
4 & $\mathrm{Pd} / \mathrm{SiO}_{2}$ & 291.00 & 134.83 & 0.98 \\
\hline
\end{tabular}

$\mathrm{C}-\mathrm{O}$ 键切断和氢转移反应上被报道过的催化剂进行尝 试 ${ }^{[24]}$, 如表 2. 结果显示, 以环己烷为溶剂, $150{ }^{\circ} \mathrm{C}$ 下反 应 $12 \mathrm{~h} \mathrm{Ni} / \mathrm{C} 、 \mathrm{Ru} / \mathrm{C} 、 \mathrm{Rh} / \mathrm{C} 、 \mathrm{Pt} / \mathrm{C}$ 对于反应几乎没有催 化效果, 而 $\mathrm{Pd} / \mathrm{C}$ 的体系得到了 $99 \%$ 的转化率, 产物苯乙 酮和愈创木酚的产率分别为 $79.8 \%$ 和 79\%, 该结果表明 $\mathrm{Pd}$ 负载型催化剂对此类反应具有良好的催化效果. 此 外, 在 $\mathrm{Pd} / \mathrm{C}$ 催化体系中, 还检测到中间体 $\mathbf{1 b}$ 生成, 产率 约为 $15.6 \%$. 这可能是脱氢过程先于 $\mathrm{C}-\mathrm{O}$ 键切断过程 导致的. 同时, 在常见的氢转移反应中, 如甲酸、异丙醇 等氢源常作为溶剂使用 ${ }^{[25]}$, 以保证提供充足的氢源, 这 说明非均相催化条件下的氢转移反应对于氢源利用

表 2 不同金属基催化剂对比优化

Table 2 The optimization of the catalysts with different metals<smiles>COc1ccccc1OCC(=O)c1ccc(-c2ccc(OC)c(OCC(O)c3ccccc3)c2)cc1</smiles>

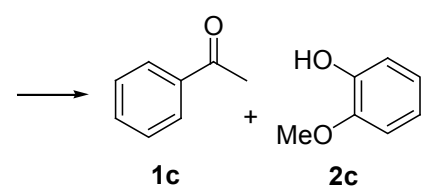

\begin{tabular}{ccccc}
\hline \multirow{2}{*}{ Cat. } & \multirow{2}{*}{ Conv. $\%$} & \multicolumn{3}{c}{ Yield $^{a} \%$} \\
\cline { 3 - 5 } & & $\mathbf{1 b}$ & $\mathbf{1 c}$ & $\mathbf{2 c}$ \\
\hline $5 \% \mathrm{Ru} / \mathrm{C}$ & 0 & - & - & - \\
$5 \% \mathrm{Ni} / \mathrm{C}$ & 0 & - & - & - \\
$5 \% \mathrm{Pd} / \mathrm{C}$ & 99 & 15.6 & 79.8 & 79.0 \\
$5 \% \mathrm{Pt} / \mathrm{C}$ & 0 & - & - & - \\
$5 \% \mathrm{Rh} / \mathrm{C}$ & 0 & - & - & - \\
\hline
\end{tabular}

$a^{a}$ 该产率数据为气相产率; 底物 $100 \mathrm{mg}$, 催化剂用量 $70 \mathrm{mg}$, 溶剂使用环己 烷 $5 \mathrm{~mL}$, 反应在 $150{ }^{\circ} \mathrm{C}$ 反应 $5 \mathrm{~h}$; 反应生成物通过 GC-MS 进行定性分析, 定量分析由 $\mathrm{GC}$ 完成.
效率相对较低. 在本反应中，仅靠分子内羟基上的氢便 可以实现模型物的高选择性解聚到单体，体现了 Pd 催 化中心针对该转化具有高的反应活性.

\section{3 反应影响因素探讨及催化剂优选}

在对催化体系的溶剂效应影响研究过程中发现，反 应溶剂的性质差异对催化剂的催化效果有较大的影响 (如图 4 所示). 溶剂极性较低的环己烷中, 反应的结果 显示转化率达到 $99 \%$, 苯乙酮和愈创木酚的产率也达到 了 $79.8 \%$ 和 $79 \%$, 在结构相似的甲苯溶剂中, 反应的转 化率为 $90.2 \%$, 愈创木酚和苯乙酮的收率分别为 $76.7 \%$ 和 75.3\%. 然而，在二甲基亚砜溶剂中，反应无法正常 进行, 检测到的转化率为 0 . 另一组对比实验显示, 四 氢呋喃溶剂中的反应达到 $16.7 \%$ 的反应转化率, 并且模 型物 $1 \mathrm{a}$ 全部转化为脱氢中间体 $\mathbf{1 b}$, 无断键产物产生. 使用环已烷四氢呋喃混合溶剂发现, 转化率较纯四氢呋 喃溶剂有明显提升，达到 $90.7 \%$ 的转化率，苯乙酮、愈 创木酚的产率也达到了 $53.6 \%$ 和 59.2\%，中间体 $\mathbf{1 b}$ 产物 收率也达到了 $35.2 \%$, 相对纯环己烷溶剂的 $11.2 \%$ 要高 很多. 根据前人的研究报道显示 ${ }^{[25,26,27]}$, 在非均相的氢 转移催化体系中, 溶剂和载体性质都起到了较大作用. 有观点认为 ${ }^{[25]}$ 由于部分溶剂分子易于与 Pd 粒子表面形 成化学吸附, 对氢原子的吸附和释放产生影响, 阻碍氢 转移的催化过程, 从而毒化催化剂, 降低催化活性.

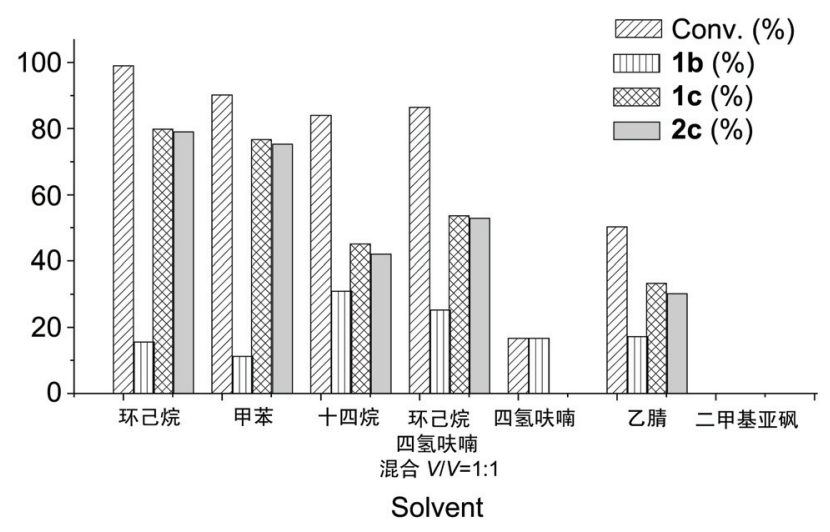

图 4 不同极性溶剂对反应影响

Figure 4 The effect of different solvents on the reaction

在相关的氢转移反应中, 反应的酸碱性也被认为是 促进反应的一个关键因素 ${ }^{[21,27]}$. 在相关的机理研究中, 研究者认为碱条件的存在有助于氢原子的拔除 ${ }^{[25,27]}$, 从 而降低产生还原性的难度. 因此, 我们研究了几种酸碱 性不同的添加剂对反应的影响. 结果显示, 碱性最强的 $\mathrm{KOH}$ 反应体系中, 转化率达到 $77.1 \%$, 而愈创木酚的收 率也较低, 只有 $16.5 \%$, 相比于苯乙酮的 $53.3 \%$ 产率要 低. 这可能因为强碱条件对弱酸性的酚类化合物有碱化 成盐的作用, 生成的酚钠难以通过 GC 检测, 致使产物 $\mathrm{GC}$ 检测产率出现偏差. 通过反应结果可以发现, 弱碱 
$\mathrm{Na}_{2} \mathrm{HPO}_{4}$ 加入对反应促进明显, 反应的转化率达到 99\%, 而苯乙酮、愈创木酚两者产率也达到了 $90.2 \%$ 和 $92.3 \%$. 值得注意的是, 其共轭酸- $-\mathrm{NaH}_{2} \mathrm{PO}_{4}$ 的效果 中, 脱氢中间体 $1 \mathrm{~b}$ 产率上升明显, 达到 $22.9 \%$. 苯乙酮 和愈创木酚的产率只有 $63.0 \%$ 和 $66.8 \%$. 这可能是 Lewis 酸含量的增加导致吸附的氢原子稳定性下降并溢出而 引发的目标产物产率下降(如表 3).

表 3 助剂对反应影响研究

Table 3 The study of additives' effect on the reaction

\begin{tabular}{ccccc}
\hline \multirow{2}{*}{ Addition $^{a}$} & Conv./\% & \multicolumn{3}{c}{ Yield $^{b} / \%$} \\
\cline { 3 - 5 } & & 1b & 1c & 2c \\
\hline $\mathrm{KOH}$ & 77.1 & trace & 53.8 & 16.5 \\
$\mathrm{NaOAc}$ & 60 & 6.0 & 58.4 & 55.6 \\
$\mathrm{NaHCO}_{3}$ & 82.3 & 4.8 & 65.4 & 61.3 \\
$\mathrm{Na}_{2} \mathrm{CO}_{3}$ & 72.8 & 6.0 & 52.9 & 52.9 \\
$\mathrm{Na}_{2} \mathrm{HPO}_{4}$ & 95.1 & 22.9 & 63.8 & 66.0 \\
$\mathrm{NaH}_{2} \mathrm{PO}_{4}$ & 99 & 6.1 & 90.2 & 92.3 \\
\hline
\end{tabular}

${ }^{a}$ 加入的添加剂以与底物 $1: 1$ 物质的量配比加入; ${ }^{b}$ 该产率数据为气相产 率.

\section{4 反应催化剂优选及过程研究}

在反应结果的检测中我们发现, $\mathrm{Pd} / \mathrm{C}$ 体系下的产物 中有少量副产物乙苯生成. 然而, 在碱性助剂加入的体 系中原有的乙基苯产物只有痕量出现. 对于这一点, 我 们认为是产物苯乙酮在反应过程中, 可能会继续夺取 $\mathrm{Pd}$ 粒子表面吸附的氢进行加氢及脱氧. 另一方面, 在诸 如 $\mathrm{NaH}_{2} \mathrm{PO}_{4}$ 酸性助剂加入后, 乙苯的产物峰有了一定的 提高, 但是在 $\mathrm{KOH}, \mathrm{Na}_{2} \mathrm{CO}_{3}$ 这样的碱性条件下, 乙苯的 生产量又降低至痕量. 由此可见, 酸性条件有利于羟基 氢解脱氧, 碱性有助于羟基脱氢. 因此, 我们考虑将催 化剂载体改为碱性, 这可能对反应的转化效率及选择性 有利.

结合添加剂对反应的影响以及前人工作 ${ }^{[25,26]}$, 在载 体篎选方面, 我们选取了常见的酸性载体、碱性载体、 高比表面载体等作为对比. 希望可以不同性质的载体探 索催化剂的催化性能变化规律. 在得到的结果中可以发 现, 常见的弱酸或酸性载体 SBA-15、HZSM-5 负载的 $\mathrm{Pd}$ 催化剂相对碱性载体 $\mathrm{MgO}$ 的催化效果要低, $\mathrm{Pd} / \mathrm{SBA}-15$ 和 $\mathrm{Pd} / \mathrm{HZSM}-5$ 的反应转化率分别只有 $78.1 \%$ 和 $80.2 \%$, 而 $\mathrm{MgO}$ 负载的催化剂反应转化率达到了 $98.0 \%$, 同时对产物苯乙酮和愈创木酚的选择性也有较 大的提高, 达到了约 $91.3 \%$ 和 90.1\%, 中间体 $\mathbf{1 b}$ 也只有 $6.0 \%$ (如表 4 所示), 这一点与之前反应体系酸碱性对反 应影响得到的结论相类似. 另一方面, 我们通过自制的 $\mathrm{Pd} / \mathrm{AC}$ 催化剂与商购的 $\mathrm{Pd} / \mathrm{C}$ 催化剂进行对比发现, $\mathrm{Pd}$ 粒子分散度对反应的影响极为重要. 自制的 $\mathrm{Pd} / \mathrm{AC}$ 在相 同反应条件下得到的苯乙酩和愈创木酚产率只有 $54.1 \%$ 和 $50.4 \%$, 结果明显差于商购 $\mathrm{Pd} / \mathrm{C}$ 的催化效果(苯乙䣶 $88.6 \%$ 、愈创木酚 $87.8 \%$ )(见表 2、表 4). 结合后期的催化 剂 TEM 表征发现, 自制 Pd/AC 的 Pd 颗粒分散性较低, 颗
粒较大, $\mathrm{Pd}$ 的粒径约为 $40 \mathrm{~nm}$ (如图 2), 在 XRD 的表征中 也出现明显的 $\mathrm{Pd}$ 晶面峰(如图 3 所示), 而商购 $\mathrm{Pd} / \mathrm{C}$ 的 TEM 和 XRD 结果都表明 Pd 颗粒相对较小(粒径约为 5 $15 \mathrm{~nm})$, 粒子的分散度也更高, 反应催化位点更多，因而 具有更高的催化活性. $\mathrm{SiO}_{2}$ 负载的 $\mathrm{Pd}$ 粒子相对也较大, 但因其载体本身的比表面较高、分散性较好，在催化效 果上优于 $\mathrm{Pd} / \mathrm{AC}$. 同时, 在催化的反应结果中检测到了 较少量的乙苯(约 4\%)生成. 弱碱性载体负载的 $\mathrm{Pd} / \mathrm{MgO}$ 催化结果中只检测到痕量的乙苯 $(<2 \%)$ 产物. 与此同 时, $\mathrm{Pd} / \mathrm{MgO}$ 也显示出较好的 $\mathrm{Pd}$ 粒子分散性和较小的 $\mathrm{Pd}$ 粒子直径(约 $20 \sim 30 \mathrm{~nm}$ ) 而表现出较好的催化效果.

表 4 不同载体的催化剂反应效果对比

Table 4 The effect of different supports of the catalyst on the reaction

\begin{tabular}{ccccc}
\hline \multirow{2}{*}{ Catalyst } & Conv./\% & \multicolumn{3}{c}{ Yield $^{a} \%$} \\
\cline { 3 - 5 } & & $\mathbf{1 b}$ & $\mathbf{1 c}$ & 2c \\
\hline $5 \% \mathrm{Pd} / \mathrm{MgO}$ & 99.0 & 6.0 & 91.3 & 90.1 \\
$5 \% \mathrm{Pd} / \mathrm{CeO}_{2}$ & 84.5 & 10.1 & 78.6 & 73.7 \\
$5 \% \mathrm{Pd} / \mathrm{TiO}_{2}$ & 68.0 & 10.7 & 52.5 & 50.6 \\
$5 \% \mathrm{Pd} / \mathrm{SiO}_{2}$ & 95.1 & 10.5 & 81.6 & 78.2 \\
$5 \% \mathrm{Pd} / \mathrm{AC}$ & 70.3 & 12.5 & 54.1 & 50.4 \\
$5 \% \mathrm{Pd} / \mathrm{SBA}-15$ & 78.1 & 16.3 & 57.7 & 55.1 \\
$5 \% \mathrm{Pd} / \mathrm{HZSM}-5$ & 80.2 & 12.5 & 64.9 & 63.9 \\
\hline
\end{tabular}

$a$ 该产率数据为气相产率.

在反应的过程方面，通过对反应过程的跟踪，我们 认为该反应过程可能分为两步(如图 5 所示): 首先通过 催化剂表面 Pd 粒子作用, 将模型物 $\mathbf{1 a}$ 芐位羟基氢拔除, 生成酮式结构中间体 $\mathbf{1 b}$, 脱去的氢原子吸附于催化剂 $\mathrm{Pd}$ 粒子表面. 其次, 完成脱氢后，酮式中间体 $\mathbf{1 b}$ 的 $\mathrm{C}-$ $\mathrm{O}$ 键在表面吸附有氢的 $\mathrm{Pd}$ 粒子催化作用下发生 $\mathrm{C}-\mathrm{O}$ 键断裂, 形成酚和苯乙酮两种产物. 副产物乙苯可能是 苯乙酮与 Pd 表面吸附的氢进一步加氢脱氧生成的产物.

在通过对反应的时间过程产物检测也能够证实上 述提出的两步反应过程. 反应时间效应显示, 反应初始, 产物苯乙酮和愈创木酚及中间体 $1 \mathbf{b}$ 均随时间增加而增 加. 反应在 $5 \mathrm{~h}$ 时, 脱氢中间体 $\mathbf{1 b}$ 产率达到最高值, 为 $15.4 \%$. 随着反应时间增加，中间体 $1 \mathrm{~b}$ 进一步降低，反 应 $12 \mathrm{~h}$ 结束后达到 $6.0 \%$, 而苯乙酠和愈创木酚含量达 到 $91.3 \% 、 90.1 \%$. 继续延长反应时间显示，苯乙酮和愈 创木酚的产率均有下降，在 $24 \mathrm{~h}$ 反应后，愈创木酚和苯 乙酩产率分别为 $75.1 \%$ 和 $78.2 \%$, 这可能是产物在反应 体系中发生了其他副反应，因而导致目标产物产率下降 (如图 6 所示).

\section{5 反应催化剂循环使用}

为了研究 $\mathrm{Pd} / \mathrm{MgO}$ 催化剂在该反应体系中的稳定性 问题, 我们通过对催化剂循环使用进行研究. 在模型反 应条件下，反应结束并冷却后，通过离心分离的方法收 集催化剂, 经溶剂乙酸乙酯进行多次冲洗除去催化剂表 面残留物, 再通过环己烷溶剂对催化剂冲洗去除残留的 


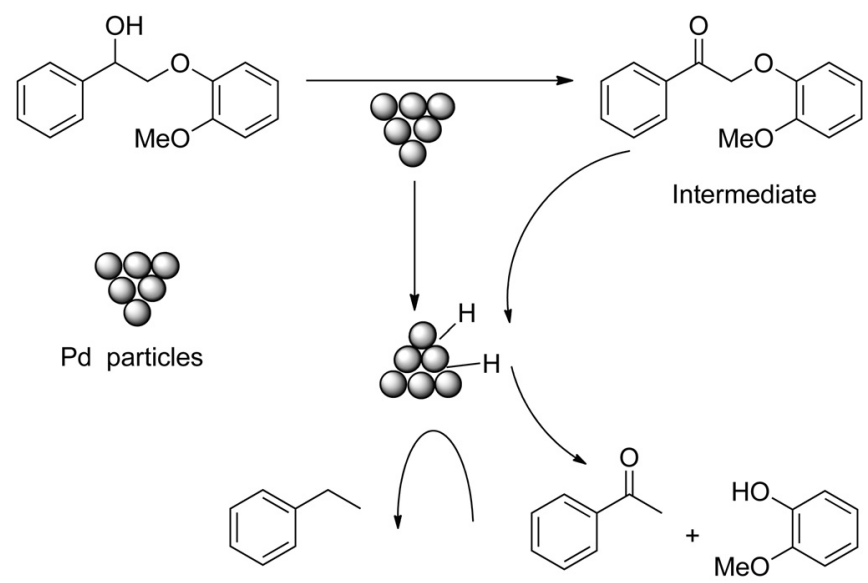

By-product

Products

图 5 木质素模型物催化条件下可能的反应过程

Figure 5 The possible process of lignin model degradation

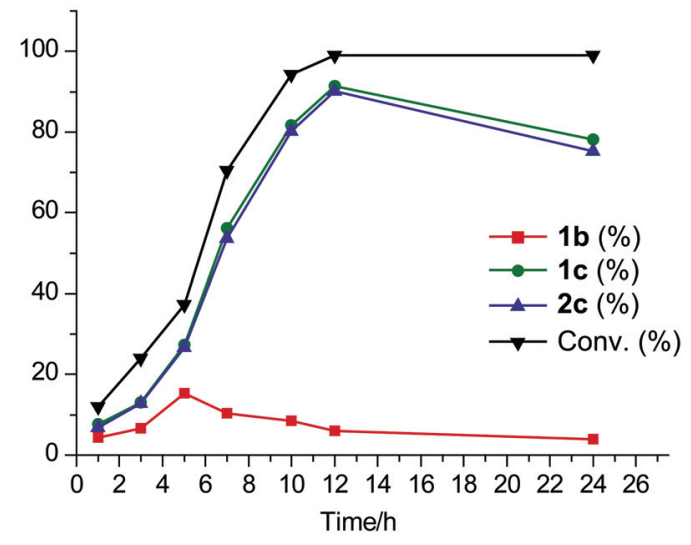

图 6 反应时间变化过程产物分布规律

Figure 6 The content of reactant and the products at different reaction time

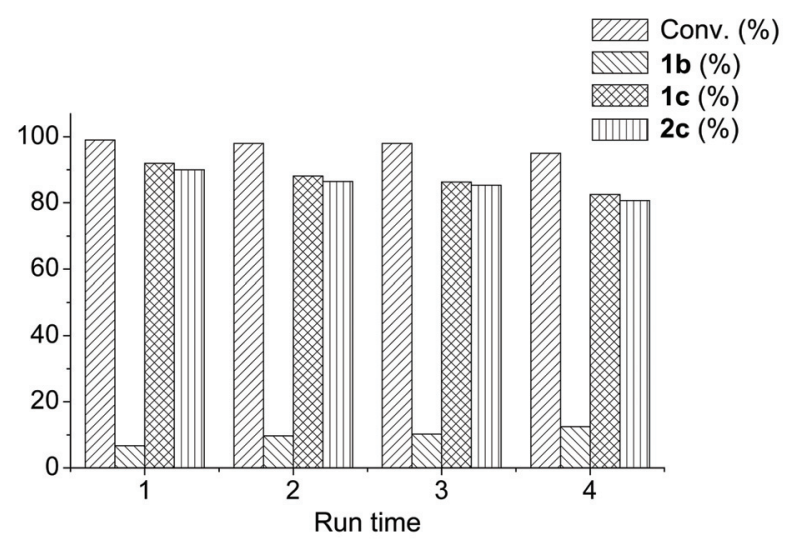

图 7 催化剂循环使用

Figure 7 The recycled using of $\mathrm{Pd} / \mathrm{MgO}$ catalyst

乙酸乙酯洗液即可进行下次循环使用. 循环反应结果如 图 7 所示, 初次反应转化率最高为 $99 \%$ 而相应的苯乙 酮、愈创木酚产率也分别达到 $92 \%$ 和 $90 \%$. 四次循环使
用后，反应转化率略有下降 $(95 \%)$, 苯乙酮和愈创木酚 产率也随之略有降低 $(82.5 \%$ 和 $80.7 \%)$. 然而, 随着循环 使用的增加，反应中间体 $1 \mathrm{~b}$ 产率呈上升趋势，这可能是 催化剂在经历高温反应后结构变化导致的活性中心钯 失活而产生的结果. 结合催化剂循环使用的实验结果证 明, $\mathrm{Pd} / \mathrm{MgO}$ 催化剂在模型反应条件下能够较好的保持 催化活性.

\section{3 结论}

Pd 系负载型催化剂对木质素二聚体模型物 $1 \mathrm{a}$ 的无 外加氢源氢解反应可行性已被证实. 我们使用 $\mathrm{Pd} / \mathrm{MgO}$ 催化剂在 $150{ }^{\circ} \mathrm{C}$ 下，经过 $12 \mathrm{~h}$ 可以将木质素模型物 1a 高效高选择性氢解至苯乙酮和愈创木酚. 本文在对反应 控制的关键因素研究中, 发现了反应体系的酸碱性不同 导致的催化效果差异. 通过对催化剂的载体调整和溶剂 体系的改变, 木质素二聚体模型物 $1 \mathrm{a}$ 在无外加氢源的 条件下通过分子内氢转移达到了较高的反应转化效率. 同时, 对整个反应的机理过程有了较深入的认识. 对比 目前各种降解木质素二聚体的催化体系来看, 分子内供 氢的氢转移降解木质素方法为降解木质素提供了一条 绿色经济的化学降解方法. 在反应高效转化的前提下, 与相类似的分子间氢转移反应相比, 无需加入过量的氢 源，对氢源的利用效率更高.

\section{4 实验部分}

$\mathrm{Pd} / \mathrm{C}(5 \% \mathrm{Pd}, \mathrm{J} \& \mathrm{~K})$; 醋酸钯 (GR, 沃凯); 活性炭 (TCI); 轻质 $\mathrm{MgO}$ (AR 级, 国药); $\mathrm{SiO}_{2}$ (气相沉淀, $99.8 \%$, 阿拉丁); $\mathrm{TiO}_{2}$ (纳米级, $99 \%$, 阿拉丁); $\mathrm{CeO}_{2}$ (纳米级, $99 \%$ ，阿拉丁); 溴代苯乙酮 $(99 \%, J \& K)$; 愈创木酚 $(98 \%$, $\mathrm{TCI})$; 环已烷(AR，国药); 甲苯(AR，国药); 四氢呋喃 (AR，国药); $\mathrm{DMSO}\left(\mathrm{AR}\right.$ ，国药); $\mathrm{KOH} 、 \mathrm{NaHCO}_{3}$ 、 $\mathrm{Na}_{2} \mathrm{CO}_{3} 、 \mathrm{NaH}_{2} \mathrm{PO}_{4} 、 \mathrm{Na}_{2} \mathrm{HPO}_{4}$ 均为( $\mathrm{AR}$, 国药). 


\section{1 催化剂制备}

使用 $100 \mathrm{~mL}$ 烧瓶, 将醋酸钯 $(0.23 \mathrm{~g})$ 用 $50 \mathrm{~mL}$ 丙酠 溶解后浸渍于载体 $(2.0 \mathrm{~g})$ 上搅拌过夜, 完成后蒸除溶剂 并置于 $120{ }^{\circ} \mathrm{C}$ 烘箱下烘干 $12 \mathrm{~h}$ 得到催化剂前体. 还原前 需要对催化剂前体进行 $550{ }^{\circ} \mathrm{C}, 8 \mathrm{~h}$ 的段烧(对比商购 $\mathrm{Pd} / \mathrm{C}$ 制备的 $\mathrm{Pd} / \mathrm{AC}$ 除外, 为了区别, 以下自制钯碳记为 $\mathrm{Pd} / \mathrm{AC}$, 商购钯碳为 $\mathrm{Pd} / \mathrm{C}$ ), 煅烧后的催化剂前体在 500 ${ }^{\circ} \mathrm{C}$ 使用 $\mathrm{H}_{2}$ 气流下还原 $5 \mathrm{~h}$.

\section{2 模型物制备}

\subsubsection{2-(2'-甲氧基苯氧基)-1-苯乙酮制备}

参考文献 ${ }^{[22]}$ 中制备方法, 使用溴代苯乙酮 $(7.0 \mathrm{~g})$ 与 愈创木酚(6.6 g) 置于 $250 \mathrm{~mL}$ 烧瓶中, 加入溶剂丙酮溶剂 $(120 \mathrm{~mL}), 80{ }^{\circ} \mathrm{C}$ 条件下经过 $\mathrm{K}_{2} \mathrm{CO}_{3}(15 \mathrm{~g})$ 反应 $5 \mathrm{~h}$. 反应 液滤除 $\mathrm{K}_{2} \mathrm{CO}_{3}$, 旋除溶剂丙酮后加入 $50 \mathrm{~mL}$ 乙醇重结晶, 产物经过乙醇重结晶冲洗并重复三次后即得到 2-(2'-甲 氧基苯氧基)-1-苯乙酮. ${ }^{1} \mathrm{H} \mathrm{NMR}\left(\mathrm{CDCl}_{3}, 400 \mathrm{MHz}\right) \delta$ : $8.01(\mathrm{~d}, J=7.5 \mathrm{~Hz}, 2 \mathrm{H}), 7.61(\mathrm{t}, J=7.5 \mathrm{~Hz}, 1 \mathrm{H}), 7.49$ (t, $J=7.5 \mathrm{~Hz}, 2 \mathrm{H}), 7.00 \sim 6.83(\mathrm{~m}, 4 \mathrm{H}), 5.36(\mathrm{~s}, 2 \mathrm{H}), 3.89(\mathrm{~s}$, $3 \mathrm{H})$.

\subsubsection{2-(2'-甲氧基苯氧基)-1-苯乙醇制备}

将木质素模型物前体 2-(2'-甲氧基苯氧基)-1-苯乙酮 $(4 \mathrm{~g})$ 溶解于 $60 \mathrm{~mL}$ 四氢呋喃-水混合溶剂中(体积比 $4: 1$ 配比). 缓慢加入 $\mathrm{NaBH}_{4}$ (2.1 g, 加入过程缓慢进行, 防 止大量气体溢出), 加入完成后室温反应 $3 \mathrm{~h}$. 完成反应 后反应液使用饱和 $\mathrm{NH}_{4} \mathrm{Cl}$ 溶液 $100 \mathrm{~mL}$ 淬灭反应, 100 $\mathrm{mL}$ 去离子水稀释溶剂再使用乙醚分次萃取反应液 $(100$ $\mathrm{mL} \times 3)$. 合并乙醚溶液并浓缩, 使用 $1 \mathrm{~mol} / \mathrm{L} \mathrm{NaOH}$ 溶 液洗涤乙醚溶液, 分离后再使用饱和 $\mathrm{NaCl}$ 溶液清洗有 机相(50 mL×3), 完成后分离有机相并使用无水硫酸钠 除水. 除去溶剂干燥得到木质素模型物 2-(2'-甲氧基苯 氧基)-1-苯乙醇. ${ }^{1} \mathrm{H} \mathrm{NMR}\left(\mathrm{CDCl}_{3}, 400 \mathrm{MHz}\right) \delta: 7.43$ (d, $J=6.8 \mathrm{~Hz}, 2 \mathrm{H}), 7.36(\mathrm{t}, J=6.8 \mathrm{~Hz}, 2 \mathrm{H}), 7.30(t, J=6.8$ $\mathrm{Hz}, 1 \mathrm{H}), 7.02 \sim 6.86(\mathrm{~m}, 4 \mathrm{H}), 5.11(\mathrm{~d}, J=9.4 \mathrm{~Hz}, 1 \mathrm{H})$, 4.17 (dd, $J=18.4,2.8 \mathrm{~Hz}, 1 \mathrm{H}), 3.98$ (t, $J=10.0 \mathrm{~Hz}, 1 \mathrm{H})$, $3.86(\mathrm{~s}, 3 \mathrm{H}), 3.33$ (s, 1H).

\section{3 实验概述}

反应称取底物 $1 \mathrm{a} 100 \mathrm{mg}$, 催化剂 $70 \mathrm{mg}$ 置于耐压 反应管中并加入溶剂环已烷 $2 \mathrm{~mL}$, 向耐压管中通入氮 气换气半分钟后封塞. 反应置于 $150{ }^{\circ} \mathrm{C}$ 油浴中磁力摚拌 反应 $12 \mathrm{~h}$. 完成后反应液使用乙酸乙酯洗取, 使用茴香 醛(98\%，阿拉丁)作为内标, GC 检测产物产率.

\section{References}

[1] Holdren, J. P. Science 2007, 315, 737.

[2] (a) Vispute, T. P.; Zhang, H.; Sanna, A.; Xiao, R.; Huber, G. W. Science 2010, 330, 1222. (b) Crossley, S.; Faria, J.; Shen, M.; Resasco, D. E. Science 2010, 327, 68. (c) Zhang, Y. H. P. J. Ind. Microbiol. Biotechnol. 2008, 35, 367 .
[3] (a) Regalbuto, J. R. Science 2009, 325, 822. (b) Holm, M. S.; Saravanamurugan, S.; Taarning, E. Science 2010, 328, 602. (c) Luo, C.; Wang, S.; Liu, H. Angew. Chem. Int. Ed. 2007, 46, 7636. (d) Mascal, M.; Nikitin, E. B. Angew. Chem. Int. Ed. 2008, 120, 8042. (e) Ragauskas, A. J.; Williams, C. K.; Davison, B. H.; Britovsek, G.; Cairney, J.; Eckert, C. A.; Frederick, Jr. W. J.; Hallett, J. P.; Leak, D. J.; Liotta, C. L.; Mielenz, J. R.; Murphy, R.; Templer, R.; Tschaplinski, T. Science 2006, 311, 484.

[4] Zakzeski, J.; Bruijnincx, P. C.; Jongerius, A. L.; Weckhuysen, B. M. Chem. Rev. 2010, 110, 3552.

[5] Chakar, F. S.; Ragauskas, A. J. Ind. Crops Prod. 2004, 20, 131.

[6] Balakshin, M. Y.; Capanema, E. A.; Chang, H. M. Characterization of Lignocellulosic Materials, Blackwell Publishing Ltd., Oxford, UK, 2008, p. 148.

[7] (a) Wang, X.; Rinaldi, R. Angew. Chem. Int. Ed. 2013, 52, 11499. (b) Wang, X.; Rinaldi, R. ChemSusChem 2012, 5, 1455. (c) Wang, X.; Rinaldi, R. Energy Environ. Sci. 2012, 5, 8244.

[8] (a) Sergeev, A. G.; Hartwig, J. F. Science 2011, 332, 439. (b) Sergeev, A. G.; Webb, J. D.; Hartwig, J. F. J. Am. Chem. Soc. 2012, 134, 20226.

[9] (a) He, J.; Zhao, C.; Lercher, J. A. J. Am Chem Soc. 2012, 134, 20768. (b) Yan, N.; Zhao, C.; Dyson, P. J.; Wang, C.; Liu, L. T.; Kou, Y. ChemSusChem 2008, 1, 626. (c) Zhao, C.; Lercher, J. A. ChemCatChem 2012, 4, 64. (d) Zhao, C.; Lercher, J. A. Angew. Chem. Int. Ed. 2012, 124, 6037.

[10] (a) Zhao, C.; Kou, Y.; Lemonidou, A. A.; Li, X.; Lercher, J. A. Angew. Chem. Int. Ed. 2009, 121, 4047. (b) Yan, N.; Zhao, C.; Dyson, P. J.; Wang, C.; Liu, L. T.; Kou, Y. ChemSusChem 2008, 1, 626. (c) Yan, N.; Yuan, Y.; Dykeman, R.; Kou, Y.; Dyson, P. J. Angew. Chem. Int. Ed. 2010, 49, 5549.

[11] Zhang, Q. S.; Wang, L. L. J. Mol. Catal (China) 2013, 27, 89. (张勤 生, 王来来, 分子催化, 2013, 27, 89.)

[12] Li, G. X.; Dong, P.; Wang, X. R.; Xu, Y. D.; Wang, C. J.; Liu, Y. J. Mol. Catal (China) 2012, 26, 26. (李贵贤, 董鹏, 王小瑞, 徐彦铎, 王成君, 刘扬, 分子催化, 2012, 26, 26.)

[13] Liang, K. Ph.D. Dissertation, University of Lanzhou, Lanzhou, 2008. (梁琨, 博士论文, 兰州大学, 兰州, 2008.)

[14] Liu, L. T.; Zhang, B.; Li, J.; Ma, D.; Kou, Y. Acta Phys.-Chim. Sin. 2012, 28, 2343. (刘凌涛, 张斌, 李晶, 马丁, 寇元, 物理化学学 报, 2012, 28, 2343.)

[15] Song, Q.; Wang, F.; Cai, J.; Wang, Y.; Zhang, J.; Yu, W.; Xu, J. Energy Environ. Sci. 2013, 6, 994.

[16] Molinari, V.; Giordano, C.; Antonietti, M.; Esposito, D. J. Am. Chem. Soc. 2014, 136, 1758.

[17] Ren, Y. L.; Yan, M.; Wang, J. J.; Zhang, Z. C.; Yao, K. S. Angew. Chem. Int. Ed. 2013, 52, 12674.

[18] Kleine, T.; Buendia, J.; Bolm, C. Green Chem. 2013, 15, 160.

[19] Desnoyer, A. N.; Fartel, B.; MacLeod, K. C.; Patrick, B. O.; Smith, K. M. Organometallics 2012, 31, 7625.

[20] (a) Nguyen, J. D.; Matsuura, B. S.; Stephenson, C. R. J. J. Am. Chem. Soc. 2014, 136, 1218. (b) Lian, Y. F.; Yan, L. L.; Wang, Y.; Qi, X. H. Acta Chim. Sinica 2014, 72, 502. (廉优芬, 间碌碌, 王羽, 漆新华，化学学报, 2014, 72, 502.)

[21] (a) Strassberger, Z.; Alberts, A. H.; Louwerse, M. J.; Tanase, S.; Rothenberg, G. Green Chem. 2013, 15, 768. (b) Zhang, J.; Teo, J.; Chen, X.; Asakura, H.; Tanaka, T.; Teramura, K.; Yan, N. ACS Catal. 2014, 4, 1574. (c) Li, C.; Zheng, M.; Wang, A.; Zhang, T. Energy Environ. Sci. 2012, 5, 6383. (d) Sturgeon, M. R.; O'Brien, M. H.; Ciesielski, P. N.; Katahira, R.; Kruger, J. S.; Chmely, S. C.; Hamlin, J.; Lawrence, K.; Glendon, B. Hunsinger, G. B.; Foust, T. D.; Baldwin, R. M.; Biddy, M. J.; Beckham G. T. Green Chem. 2014, 16, 824. (e) Zhou, X.; Mitra, J.; Rauchfuss, T. B. ChemSusChem 2014, 7, 1623. (f) Harms, R. G.; Markovits, I. I.; Drees, M.; Herrmann, W. A.; Cokoja, M.; Kühn, F. E. ChemSusChem 2014, 7, 429.

[22] Nichols, J. M.; Bishop, L. M.; Bergman, R. G.; Ellman, J. A. J. Am. Chem. Soc. 2010, 132, 12554.

[23] Guo, J. L.; Shen, Y. N. Chinese J. Inner Mongolia Normal University 2009, 38, 357. (郭金玲, 沈岳年, 内蒙古师范大学学报, 2009, 


\section{$38,357$.}

[24] (a) Tanner, D.; Somfai, P. Tetrahedron 1987, 43, 4395. (b) Jae, J.; Zheng, W.; Lobo, R. F.; Vlachos, D. G. ChemSusChem 2013, 6, 1158.

[25] Johnstone, R. A. W.; Wilby, A. H.; Entwistle, I. D. Chem. Rev. 1985, 85,12 .
[26] Huang, L.; Zhu, Y.; Huo, C.; Zheng, H.; Feng, G.; Zhang, C.; Li, Y J. Mol. Catal. A: Chem. 2008, 288, 109.

[27] Zhang, T.; Tan, H. J.; Hong, Y. L.; Shen, L. J. Hangzhou Normal University (Nat. Sci. Ed.) 2012, 11, 7. (张添, 谭华杰, 洪益玲, 沈 良, 杭州师范大学学报: 自然科学版, 2012, 11, 7.)

(Cheng, B.) 